\title{
Target-rate Tracking for Shale-gas Multi-well Pads by Scheduled Shut-ins *
}

\author{
Brage R. Knudsen* Bjarne Foss * Curtis H. Whitson ** \\ Andrew R. Conn *** \\ * Department of Engineering Cybernetics, Norwegian University of \\ Science and Technology, Trondheim, Norway. \\ ** Department of Petroleum Engineering 6 Applied Geophysics, \\ Norwegian University of Science and Technology, Trondheim, Norway. \\ *** IBM T. J. Watson Research Center, Yorktown Heights, NY, USA.
}

\begin{abstract}
The recent success of shale-gas production relies on drilling of long horizontal wells and stimulation with multistage hydraulic fracturing. This practice normally leads to an initial peak production with a subsequent rate decline, followed by low and erratic production rates caused by water accumulation in the wells. Shale-gas recovery requires a large number of wells in order to maintain a sustainable total gas supply. To reduce the surface area disturbances caused by this extensive drilling and to share available surface infrastructure, the use of multi-well pads is a key driver in shale-gas developments. Furthermore, the inherent rate decline of shale-gas wells, the water accumulation in them and the large number of wells, leads to severe operational challenges for well operators. The fact that shut-ins may be used as a means to prevent liquid loading and boost late-life production rates from shale-gas wells, suggests scheduling of shut-ins to perform maintenance and clean-up of the wells, and to track a target rate for the multi-well pad. In this paper we propose an optimization scheme for shale-gas multi-well pads to schedule shut-ins and to track a target rate. The optimization problem is formulated as a discrete time mixed integer linear program (MILP) with binary variables defining at which times the well is either shut-in or producing. A reservoir proxy model and a well model for each well is designed and tuned against a realistic multi-fractured reservoir model. We demonstrate the benefits and the potential of the proposed methodology through a one-month production planning problem for an eight-well shale-gas pad.
\end{abstract}

Keywords: Optimization, Scheduling, Reduced-order models, Petroleum production, Plantwide control.

\section{INTRODUCTION}

Organic rich, ultra-tight shale rock may store enormous amounts of natural gas. Shale gas as an energy resource is growing rapidly and currently constitutes more than $20 \%$ of the drilled gas production in the U.S [Sutton et al., 2010]. The inherently low permeability of shale, however, makes extraction of the gas very challenging. The generation of conductive interconnected fractures by multiple stimulations with hydraulic fracturing $(\mathrm{HF})$ is therefore crucial for draining reasonable amounts of gas from the low permeable shale. Horizontal wells together with multistage hydraulic fracturing is by far the most common completion technique in current shale-gas developments [Cipolla et al., 2009]. These stimulation treatments create a network of fractures, the size and shape of which depend on the extent of preexisting fracture networks in the shale, the conditions of the hydraulic fracturing and the stress principles in the shale rock.

Exploitation of shale gas is land-based, and normally requires a very large number of wells to achieve profitable

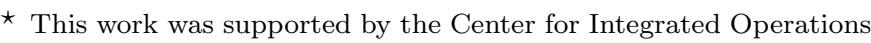
in the Petroleum Industry, Trondheim, Norway.
}

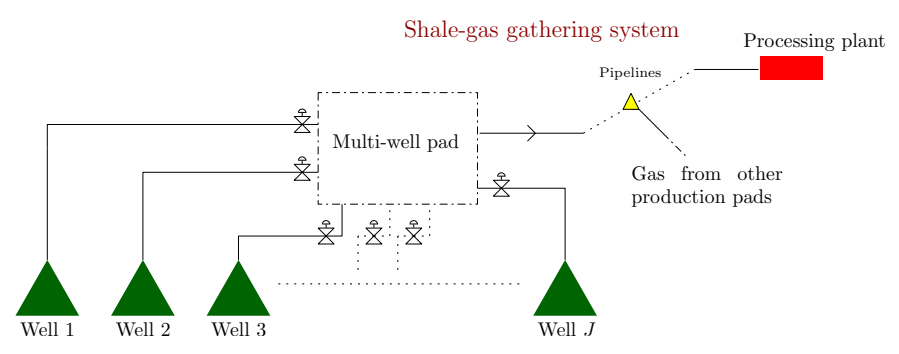

Fig. 1. Illustration of shale-gas surface production systems.

recovery rates. Traditionally, the wells are widely spread over a large geographical area. The current trend is towards multi-well pads, both to share surface infrastructure and to reduce the footprint [Stefik and Paulson, 2011]. Dry and semi-dry shale-gas wells exhibit many of the same characteristics: an early peak in the gas rate from the sudden release of gas stored in pores and natural fracture networks, followed by a long transient decline in the production rate. The rate decline is caused both by pressure depletion and the inherently low permeability of shales, but in many cases also by liquid accumulation in the well from low saturations of water entrapped in the extracted gas. The liquid accumulation increases the 
back pressure on the vertical parts of the well, and as the reservoir pressure decreases, the wells' ability to lift out loaded water will be reduced. Eventually the well will exhibit liquid loading recognized by erratic and unstable rates [Al Ahmadi et al., 2010, Sutton et al., 2010], requiring shut-ins to clean-up the well.

The continual drilling and enormous amount of wells required to offset the field's rate decline results in a severe and challenging control problem for the operators. A typical structure of shale-gas surface production systems is illustrated in Fig. 1. Single and multi-well pads are connected by comprehensive pipeline networks which transports the gas to midstream stations or processing plants. Frequent shut-ins of the distributed wells may be necessary, both for clean-ups but also for production tests and fracturing jobs at nearby wells. The operators on midstream stations may be required to provide a certain rate over a given time horizon, either to gas compressors or processing plants, or directly to the market transmission lines. Consequently, avoiding sudden simultaneous shut-ins is desirable both to maintain the pads' gas deliverability and thereby produce according to a preset production plan, and to minimize variations in the production line pressure. In addition, there are only few ways to control or influence shale-gas well production and thereby prevent the rate decline.

In this paper we propose a scheme to optimize mid or late-life performance of shale-gas wells by using shut-ins, and we show how the shut-ins can be efficiently scheduled to meet target rates and to perform maintenance and clean-ups of the wells. To solve the scheduling problem we formulate a mixed-integer linear program (MILP), using a proxy model of the well and the reservoir which is tuned against a full scale simulator model. The article is organized as follows: section 2 describes the reservoir and well proxy model and the tuning against a high-fidelity reference model. The MILP formulation is presented in section 3, and numerical results are shown in section 4 . Finally, section 5 discusses the problem formulation and its applicability while a conclusion ends the paper.

\section{SHALE-GAS RESERVOIR MODELING}

Gas is stored in shales both as free gas in the fractures, in the porous space of the shales and as absorbed gas within the organic material. The gas is released by high pressure gradients, and by interaction between the highly conductive fractures and the low permeable shale matrix. Fractured shale-gas reservoirs can be modeled either by the dual-porosity idealization [Warren and Root, 1963], as discrete fracture models [Karimi-Farad et al., 2004], or as fully discretized dual-permeability models [Cipolla et al., 2009]. All these modeling schemes have different merits and drawbacks in terms of accuracy, complexity, numerical efficiency and the ability to match production data. The model used in this paper is a single-porosity, dualpermeability model with cylindrical geometry as sketched in Fig. 2. The gas is assumed to be dry (single phase) and the fracture conductivity is assumed to be finite. Fracture unconsolidation, i.e. reduction of permeability and porosity when pressure reduces, is not included. Gas desorption from the organic material may for some shale-gas wells be a significant part of the long-term recovery. The impact of gas desorption is however primarily observable later on, typically beyond 10 years and may then constitute $5-15 \%$ of the total gas production in 30 years [Cipolla et al., 2009], depending on the matrix permeability and the primary fracture spacing. The time scale in the subsequent simulations are limited to 1-5 years. This justifies the omittance of gas desorption in the model.

\subsection{Analytical proxy model}

Proxy models are often necessary to solve nonlinear (nonconvex) optimization problems. Proxy models always represent a trade-off between accuracy, complexity and numerical efficiency. The proxy model applied in this work is constructed as a single-layered, radial composite reservoir model as illustrated in Fig. 2. Assuming Darcys law, using the law of mass conservation and the equation of state for real gases, the basic nonlinear partial differential equation (PDE) for the gas flow in the radial proxy model is

$$
\phi \frac{p}{Z(p)} c(p) \frac{\partial p}{\partial t}=\frac{1}{r} \frac{\partial}{\partial r}\left(k(r) \frac{p}{\mu(p) Z(p)} r \frac{\partial p}{\partial r}\right),
$$

where $p$ is the pressure, $k(r)$ is a radially dependent permeability, $\mu(p)$ is the gas viscosity, $Z(p)$ is the gas compressibility factor and $c(p)$ is the total compressibility. Assuming isothermal conditions and the natural condition that $c_{g} \gg c_{t}$, where $c_{g}$ is gas compressibility and $c_{t}$ is rock compressibility, then

$$
c(p)=\left.\frac{1}{\rho} \frac{\partial \rho}{\partial p}\right|_{T}=\frac{1}{p}-\left.\frac{1}{Z} \frac{\partial Z}{\partial p}\right|_{T},
$$

where $\rho$ is gas density. The pressure dependency of $\mu(p)$

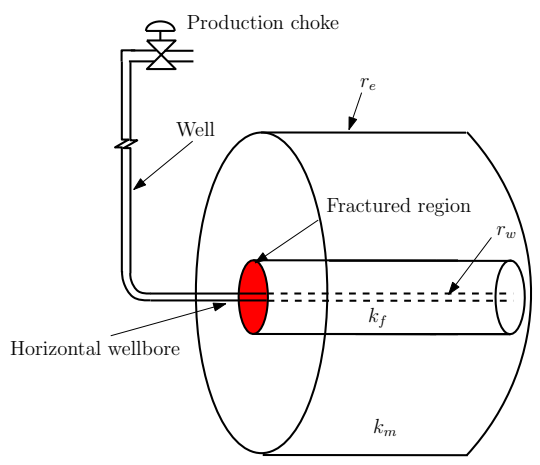

Fig. 2. Illustration of proxy model

and $Z(p)$ can be efficiently handled by the variable transformation known as pseudopressure [Al-Hussainy et al., 1966],

Since

$$
m(p)=2 \int_{0}^{p} \frac{p^{\prime}}{\mu\left(p^{\prime}\right) Z\left(p^{\prime}\right)} d p^{\prime} .
$$

$$
\frac{\partial m}{\partial t}=\frac{\partial m}{\partial p} \frac{\partial p}{\partial t}=2 \frac{p}{\mu Z} \frac{\partial p}{\partial t},
$$

and $\partial m / \partial r$ can be obtained similarly, (1) is reformulated in terms of pseudopressure $m(p)$ as

$$
\phi \mu(p) c(p) \frac{\partial m}{\partial t}=\frac{1}{r} \frac{\partial}{\partial r}\left(k(r) r \frac{\partial m}{\partial r}\right) .
$$

By using Neumann boundary conditions with a producing well (sink) in the center and no-flow conditions at the 
outer boundary, the initial-boundary value problem for the reservoir gas flow may be summarized as follows:

$$
\begin{aligned}
\phi \mu c \frac{\partial m}{\partial t} & =\frac{1}{r} \frac{\partial}{\partial r}\left(k(r) r \frac{\partial m}{\partial r}\right), \\
\left.r \frac{\partial m}{\partial r}\right|_{r_{w}} & =q \frac{T p_{s c}}{T_{s c} \pi h k}, \\
\left.\frac{\partial m}{\partial r}\right|_{r_{e}} & =0, \\
m(r, 0) & =m_{i} .
\end{aligned}
$$

By evaluating the compressibility at a given pressure, and hence treating the product $\mu(p) c(p)$ as a constant, (6) is a semi-linear PDE. The initial pseudopressure is calculated by trapezoidal integration of the corresponding values of $p, \mu$ and $Z$ which are obtained by gas correlations. Consequently, all reservoir dynamics are calculated in terms of pseudopressure $m(p)$ instead of pressure $p$. In the subsequent section, the pressure dependency of the product $\mu c$ in (6) is omitted.

\subsection{Discretization}

The PDE (6) is discretized using a finite difference scheme. Using central difference approximations for the spatial derivatives, the semi-discretization of (6) for grid block $i$ yields

$$
\phi \mu c \frac{\partial m_{i}}{\partial t}=\frac{r_{i+\frac{1}{2}} k_{i+\frac{1}{2}}\left(\frac{m_{i+1}-m_{i}}{r_{i+1}-r_{i}}\right)-r_{i-\frac{1}{2}} k_{i-\frac{1}{2}}\left(\frac{m_{i}-m_{i-1}}{r_{i}-r_{i-1}}\right)}{r_{i}\left(r_{i+\frac{1}{2}}-r_{i-\frac{1}{2}}\right)} .
$$

Multiplying both sides of (10) with $r_{i}\left(r_{i+\frac{1}{2}}-r_{i-\frac{1}{2}}\right) \frac{\pi h T_{s c}}{T p_{s c}}$ gives the left hand side

$$
\phi \mu c r_{i}\left(r_{i+\frac{1}{2}}-r_{i-\frac{1}{2}}\right) \frac{\pi h T_{s c}}{T p_{s c}} \frac{\partial m_{i}}{\partial t}
$$

and the right hand side

$$
\begin{aligned}
\underbrace{\frac{\pi h r_{i+\frac{1}{2}} k_{i+\frac{1}{2}} T_{s c}}{\left(r_{i+1}-r_{i}\right) T p_{s c}}\left(m_{i+1}-m_{i}\right)}_{-q_{i+\frac{1}{2}}} & \underbrace{\frac{\pi h r_{i-\frac{1}{2}} k_{i-\frac{1}{2}} T_{s c}}{\left(r_{i}-r_{i-1}\right) T p_{s c}}\left(m_{i}-m_{i-1}\right)}_{-q_{i-\frac{1}{2}}},
\end{aligned}
$$

where $q_{i-\frac{1}{2}}$ and $q_{i+\frac{1}{2}}$ are the gas flow in and out of grid block $i$ in surface rates, respectively. The close wellbore dynamics will be significantly faster than the dynamics in the low permeability outer region, and it also dominates the well inflow from the reservoir. A discretization scheme with irregular grid spacings will therefore be applied to improve the accuracy of the discretized PDE (11) and (12). The stationary solution of equation (6) with homogeneous properties gives a pseudopressure drop between successive grid blocks linear in $\ln r$. This property is used in choosing the irregular spacing of the central grid co-ordinates. Hence

$$
\frac{r_{i+1}}{r_{i}}:=\xi,
$$

with $\xi$ constant. Consequently, the grid block co-ordinates will increase in a geometric progression with $\xi$ as the geometric factor and $r_{i}=\xi^{i-1} r_{1}$. Given $N_{r}$ radial grid points, the expressions for $r_{1}$ and $\xi$ are obtained by requiring $r_{e}$ to be the logarithmic mean of $r_{N_{r}}$ and $r_{N_{r}+1}$, and $r_{w}$ to be the logarithmic mean of $r_{0}$ and $r_{1}$. This gives

$$
\xi=\left(\frac{r_{e}}{r_{w}}\right)^{\left(1 / N_{r}\right)}
$$

and

$$
r_{1}=r_{w} \frac{\xi \ln \xi}{\xi-1} .
$$

Each of the two terms in (12) are discretized Darcy flow rates in and out of grid block $i$ expressed in surface rates $\mathrm{Sm}^{3} / \mathrm{s}$. These flow rates will be identical to the exact Darcy flow rates by choosing the grid block boundaries $r_{i \pm \frac{1}{2}}$ in the transmissibility calculations in (12) as the logarithmic mean of $r_{i}$ and $r_{i+1}$,

$$
r_{i+\frac{1}{2}}^{L}=\frac{r_{i+1}-r_{i}}{\ln \frac{r_{i+1}}{r_{i}}} .
$$

Equation (11) represents the gas volume accumulation in each grid block, and can be rewritten

$$
\begin{aligned}
& \phi \mu c \frac{T_{s c}}{2 T p_{s c}} V_{i}^{d} \frac{\partial m_{i}}{\partial t}, \\
& V_{i}^{d}:=2 \pi r_{i}\left(r_{i+\frac{1}{2}}-r_{i-\frac{1}{2}}\right) h,
\end{aligned}
$$

where $V_{i}^{d}$ is the discretized volume of grid block $i$. Using the block boundaries (16) in (18) leads to discretized block volumes $V_{i}^{d}$ different from the actual volumes of the grid blocks. However, by substituting $s:=r^{2}$ in (6) and using the same difference approximations, it can be shown that the grid block boundaries for the discretized block volumes (18) should be the logarithmic mean of $r_{i}^{2}$ and $r_{i+1}^{2}$, while the block boundaries in the transmissibilities should be calculated from (16). See Abou-Kassem et al. [2006] or Aziz and Settari [1979] for more details on the discretization scheme.

The time discretization of (6) is obtained by using the backward Euler scheme. An implicit scheme is used in order to secure stability of the model. This leads to the discretized reservoir model

$$
\begin{aligned}
\mathbf{A} \mathbf{m}_{k+1} & =\mathbf{m}_{k}+\mathbf{B} q_{k+1} \\
\mathbf{m}_{0} & =\mathbf{m}_{\text {init }},
\end{aligned}
$$

where $\mathbf{m}$ is a vector containing the grid pseudopressures in each grid block, $\mathbf{A}$ is a tridiagonal matrix and $q$ is the scalar gas rate flowing into the well.

\subsection{Well model}

The flow rate $q$ is normally controlled indirectly through the well's bottomhole pressure $p_{w f}$, or through the production choke opening, incorporated into the well model. The coupling between the well and the reservoir depends on the well configuration and the type of well constraints. Nevertheless, the coupling should represent the essential expected features of the well performance: the well may deliver a constant rate for a short time, while a constant flowing bottomhole pressure may lead to an unrealistic high initial rate. Usually, a desired rate is specified with constraints on the flowing bottomhole pressure [AbouKassem et al., 2006], derived from surface boundary conditions such as minimum manifold or wellhead pressure. To 
convert such surface constraints to bottomhole pressures, one common practice in reservoir simulations is to specify a constant wellhead pressure and connect the reservoir model to a steady-state wellbore model derived using the mechanical energy balance. For gas wells, the steady-state wellbore model is a 2 nd order polynomial of the rate and the wellhead/bottomhole pressure [Katz and Lee, 1990]. This would require a nonlinear mapping to pseudopressure $m(p)$ when connected to the reservoir model. To circumvent this problem and to include the basic features of the well performance described above, we use the assembled well and wellbore model

$$
q_{k}=\min \left\{Q^{\max }, \alpha_{k} w\left(m_{1, k}-m\left(e^{s} p_{w}\right)\right)\right\},
$$

where $Q^{\max }$ is the desired rate, $w$ is a skin dependent well index, $m_{1, k}$ is the pseudopressure in the innermost grid block, $p_{w}$ is a specified constant wellhead pressure and $\alpha_{k} \in[0,1]$ is the production choke opening. The well will initially deliver a constant rate during which the bottomhole pressure can be post calculated. Once the well is unable to deliver the constant rate $Q^{\max }$, the rate starts to decline. The maximum rate compensates for the model's lack of pressure drop due to wellbore friction, while the term $e^{s} p_{w}$ yields the static column pressure. See Katz and Lee [1990].

\subsection{Model tuning}

The proxy model in 2.2 is tuned against a multi-frac horizontal reservoir model illustrated in Fig. 3. The multifrac model is constructed with 10 individual fractures, where each fracture is perpendicular to the horizontal wellbore. This model represents a realistic shale-gas reservoir and fracture geometry after multiple sequential stimulations with hydraulic fracturing. The high-fidelity multifrac model is implemented in the state-of-the-art simulation software SENSOR ${ }^{1}$ using logarithmic grid refinements around the fractures and more than 2500 grid cells.

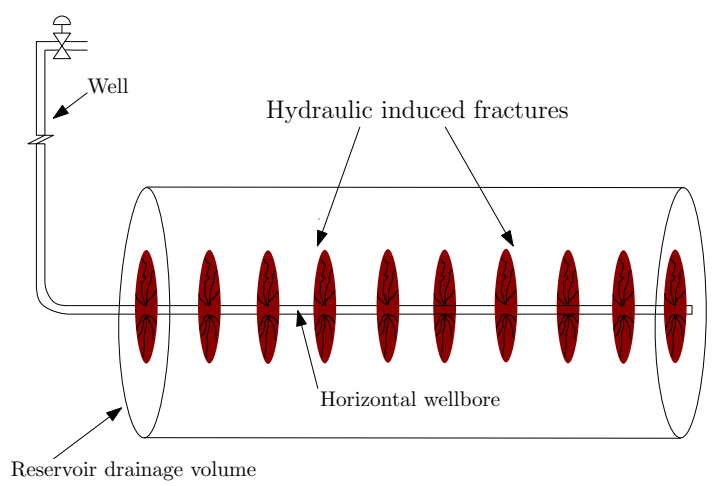

Fig. 3. Illustration of the multi-frac horizontal shalegas reservoir model implemented in the reservoir simulation software SENSOR.

The objective of the proxy model is 1) to capture the dominating dynamics during shut-ins and re-openings, and 2) to be computationally simple enough to be included in a full-space optimization problem without sacrificing accuracy in the frequency range of interest. Hence, there is a trade-off between matching the model initially with fast pressure dynamics and depletion of the fracture volume, and matching the reservoir pressure build-up during shutins. The latter is emphasized since the model is used to optimize shut-ins at late times. The proxy model is constructed with three concentric regions with distinct properties. Between the low permeable outer region and the high permeable inner region, representing the shale matrix and the fractures respectively, we consider an intermediate transition region with average properties of these two regions. The grid in the proxy model is constructed using totally $N_{r}=12$ radial grid blocks.

The tuned parameters in the proxy model are the horizontal well length, the permeability in the transition region and the constant compressibilities in the three regions. The radial and the fracture extent are equal for the two models. By minimizing time-varying sum of squares (SSQ), the proxy model is tuned over a 70 day time horizon from 295 to 365 days after the start-up, using two predefined shutins on one week and ten days, respectively. To solve the parameter estimation problem, a mesh-adaptive direct search algorithm is used due to the discontinuous derivatives of the well model (20). The SSQ weights are higher in the two regions of decline after the shut-ins than during the shutin periods and the pseudo steady-state rate. From Fig. 4 we observe that the proxy model matches the reference model well in the regions of decline, particularly during the decline after the one-week shut-in at $t=300$ days. The proxy model misses out on some of the curvature from the reservoir pressure drop next to the ten-day shut-in. This may indicate that the proxy model better matches short shut-ins than long shut-ins. Since the SSQ is weighted in the regions of the rate decline, there will be a discrepancy in the pseudo steady-state response. The transition region is mainly to remedy the numerical problems related to the large difference in permeability magnitude between the two regions $\left(\sim 10^{6}\right)$. On the other hand, the physical transition between the fractures and the shale matrix is unlikely to be as abrupt as represented in a pure dualpermeability model.

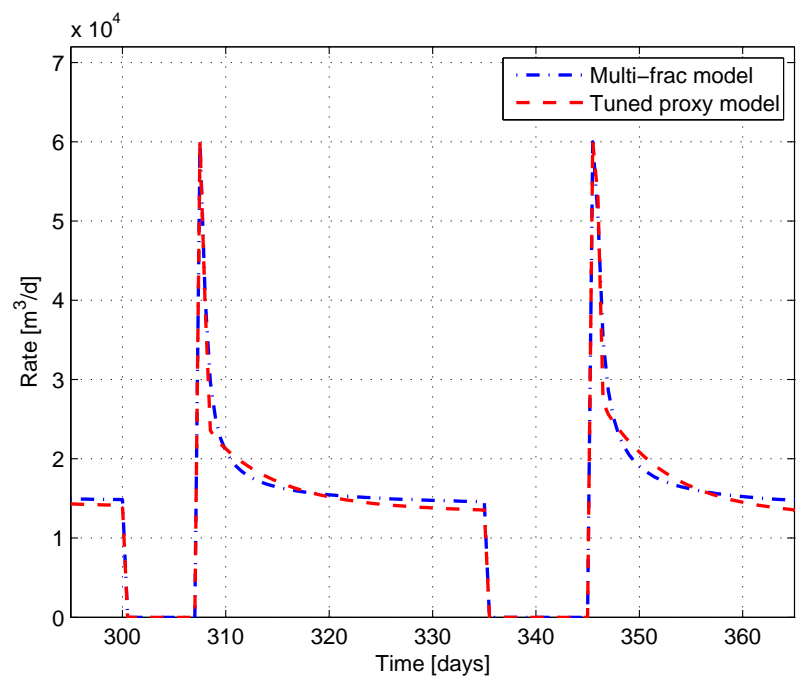

Fig. 4. Tuning of the proxy model against the high-fidelity (reference) model illustrated in Fig. 3.

1 Coats Engineering, Inc. 


\section{FORMALIZING THE OPTIMIZATION PROBLEM}

The optimization problem is formulated as a scheduling problem for a single multi-well pad based on the criteria discussed in the introduction, i.e. meeting a target rate and avoiding well liquid loading by scheduling individual well shut-ins. To model the on/off state of the wells from the shut-ins, the control variables are the production choke openings in (20), defined as binary variables $\alpha_{k} \in\{0,1\}$. The reservoir and well models (19)-(20) will be included as explicit constraints in the optimization problem, with an exact linear reformulation of (20). Hence, to exploit the efficiency of MILP solvers, the conventional quadratic objective function in tracking problems is replaced by the linear objective function

$$
\max \sum_{k=0}^{N}\left[\sum_{j=1}^{J}\left(G_{p} q_{k}^{j}\right)-\mu_{1} q_{k}^{s}-\mu_{2} q_{k}^{u}\right] \Delta k,
$$

with the constraints

$$
\begin{array}{ll}
Q^{\text {tot }, k}=\sum_{j=1}^{J}\left(q_{k}^{j}\right)-q_{k}^{s}+q_{k}^{u}, & k=0, . ., N \\
q_{k}^{s}, q_{k}^{u} \geq 0, & k=0, . ., N
\end{array}
$$

where $G_{p}$ is the gas price, $N$ is the number of time steps, $Q^{t o t, k}$ is the target rate, $q_{k}^{s}$ and $q_{k}^{u}$ are the per-time-step surplus and underproduction for the $J$ wells, respectively, and $\mu_{1}$ and $\mu_{2}$ are nonnegative penalty parameters. There are several advantages of using the linear formulation (21)-(22) instead of a mixed integer quadratic problem (MIQP): the MILP solvers are generally more efficient in terms of hot-starts and re-use of basis for successive nodes in an enumeration scheme (i.e., branch-and-bound and branch-and-cut). Secondly, (21) retains an economical interpretation as the penalty parameters may be selected based on the specifications in a gas sales contract.

\subsection{Reformulation of nonlinearities}

The well model (20) has discontinuous first order derivatives and is unfavorable as a constraint in any optimization problem. However, since the nonlinearity consists of the $\min (\cdot)$ function and the bilinear product of the binary shut-in variables $\alpha_{k}$ the continuous variables $m_{1, k},(20)$ can be reformulated exactly to a set of linear constraints. Let the second term in (20) be expressed as

$$
\begin{aligned}
\tilde{q}_{k}: & =\tilde{q}_{k}^{n l}-\alpha_{k} w m\left(e^{s} p_{w}\right), \\
\tilde{q}_{k}^{n l}: & =\alpha_{k} w m_{1, k},
\end{aligned}
$$

and let $M^{\max }$ be the least upper bound of the grid pseudopressures for all time steps $k$. The nonlinear well model (20) is then equivalent with the linear constraints

$$
\begin{aligned}
\tilde{q}_{k}^{n l} & \geq \alpha_{k} w m\left(e^{s} p_{w}\right), \\
\tilde{q}_{k}^{n l} & \leq \alpha_{k} w M^{\max }, \\
w m_{1, k}-\tilde{q}_{k}^{n l} & \geq w m\left(e^{s} p_{w}\right)\left(1-\alpha_{k}\right), \\
w m_{1, k}-\tilde{q}_{k}^{n l} & \leq w M^{\max }\left(1-\alpha_{k}\right), \\
\tilde{q}_{k} & =\tilde{q}_{k}^{n l}-\alpha_{k} w m\left(e^{s} p_{w}\right), \\
q_{k} & \leq Q^{\max }, \\
q_{k} & \leq \tilde{q}_{k}, \\
q_{k} & \geq Q^{\max } d_{k}, \\
q_{k} & \geq \tilde{q}_{k}-w\left(M^{\max }-m\left(e^{s} p_{w}\right)\right) d_{k},
\end{aligned}
$$

where $d_{k} \in\{0,1\}$. Note that the well index is omitted, while the reformulation of the disjunctive constraint (20) is performed for all wells $j$ for all time steps $k$.

\subsection{Critical flow rate}

Turner et al. [1969] proposed an estimate for the critical gas velocity needed to ensure continuous lift of liquid droplets in the tubing. The model is based on a droplet suspended in the flowing gas, and estimates the critical velocity by

$$
v_{g c}=c \frac{\left[\sigma\left(\rho_{l}-\rho\right)\right]^{0.25}}{\rho^{0.5}},
$$

where $\rho_{l}$ is the liquid density and $\sigma$ is the interfacial tension. Turner et al. [1969] suggested the value $c=6.2$ (SI-units), while several later studies have observed the estimate to be conservative and not necessarily reliable. Notwithstanding, the estimate is accepted to be a good criterion for the onset of liquid loading in gas wells. Onset of liquid loading is dominated by the wellhead conditions [Turner et al., 1969]. Consequently, the temperature, the compressibility factor and the pressure are necessary evaluated at wellhead input conditions. Using the real gas law and assuming $\sigma=0.06 \mathrm{~N} / \mathrm{m}^{2}$ and $\rho_{l}=1074 \mathrm{~kg} / \mathrm{m}^{3}$ [Turner et al., 1969], the critical rate (the Turner rate) is given by

$$
\begin{aligned}
& v_{g c}\left(p_{w}\right)=6.2 \frac{\left[\sigma\left(\rho_{l}-\kappa p_{w}\right)\right]^{0.25}}{\kappa p_{w}^{0.5}}, \quad \kappa:=\frac{G M_{a i r}}{Z_{w} R T_{w}}, \\
& q_{g c}\left(p_{w}\right)=8.64 \cdot 10^{4} A_{t} \frac{p_{w} T_{s c}}{p_{s c} T_{w} Z_{w}} v_{g c}\left(p_{w}\right),
\end{aligned}
$$

where $G$ is the gas specific gravity, $M_{\text {air }}$ is the molecular weight of air and $A_{t}$ is the cross-section area of the tubing. Below the critical rate, liquid droplets may fall out and accumulate in the tubing. The Turner rate will therefore be imposed as a flow rate constraint and used as necessary criterion to shut in the wells. However, the constraint is disjunctive and must be relaxed each time the well is shut in. This is handled by formulating the lower bound on the flow rate as the constraint [Floudas, 1995]

$$
q_{g c}\left(p_{w}\right) \alpha_{k} \leq q_{k} .
$$

The constraint (28) makes it possible, without including water saturation in the reservoir model, to predict at which rate and at which time a well must be shut in to avoid liquid loading.

\subsection{Operational constraints}

Each well is assumed to operate at a constant wellhead pressure, and the common production constraints are thus from the target rate (22). Care should be taken regarding the number and duration of well shut-ins. There are several reasons for this. Frequent shut-ins may damage the equipment, the operators need time to perform necessary maintenance and clean-up during shut-ins, and each shutin may have to be performed over a certain time to allow pressure equalization and to avoid abrupt pressure drops. Auxiliary variables $\eta_{k} \in \mathbb{R}$ are introduced for counting the number of well switchings. The constraints

$$
\left|\alpha_{k+1}-\alpha_{k}\right| \leq \eta_{k+1},
$$

enforce $\eta_{k}$ to one each time the well is either shut-in or reopened. Reformulating (29) as linear constraints is trivial. 
The parameter $\tau$ is introduced as a tuning parameter that determines for how long, at a minimum, a well must be shut-in each time it is closed. This together with the constraints

$$
\sum_{i=k+1}^{k+\tau-1} \eta_{i} \leq\left(1-\eta_{k}\right), \quad k=0, . ., N-\tau-1,
$$

ensures that the well is closed at least $\tau$ time steps each time it is shut in.

\section{NUMERICAL EXAMPLE}

Under the model assumptions, the optimization problem is formulated as an MILP and implemented in the software Xpress-IVE from FICO ${ }^{\mathrm{TM}}$. The presented approach is applied to a numerical example using a multi-well pad with eight wells and a planning horizon of four weeks. Since the shale-gas wells are drilled and completed sequentially, each well on the pad is assumed to have been operational for different lengths of time and hence have different initial conditions. Parameter values used in the example are all realistic and are given in Table 1 . We choose

Table 1. Parameter values.

\begin{tabular}{llll}
\hline Symbol & Parameter & Value & Unit \\
\hline$\phi$ & porosity & 6 & $\%$ \\
$k_{m}$ & matrix permeability & $3.5 \times 10^{-4}$ & $\mathrm{mD}$ \\
$k_{f}$ & fracture permeability & 100 & $\mathrm{mD}$ \\
$z_{w}$ & true vertical depth & 2300 & $\mathrm{~m}$ \\
$r_{e}$ & radial extent & 564 & $\mathrm{~m}$ \\
$p_{r}$ & initial reservoir pressure & $2 \times 10^{7}$ & $\mathrm{~Pa}$ \\
$p_{w}$ & wellhead pressure & $1 \times 10^{6}$ & $\mathrm{~Pa}$ \\
$Q^{\max }$ & maximum rate & $6 \times 10^{4}$ & $\mathrm{~m}^{3} / \mathrm{d}$ \\
$\Delta k$ & fixed time step & 1 & days \\
$T$ & prediction horizon & 28 & days \\
$J$ & number of wells & 8 & - \\
$\tau$ & minimum shut-in time & 5 & days \\
\hline
\end{tabular}

the values $\mu_{1}=\mu_{2}=2 G_{p}$ for the penalty parameters with the purpose of achieving as tight target-rate tracking as possible. These values of the penalty parameters are therefore not necessarily related to the loss in revenue from too little total production or surplus gas. Using eight wells, a one day time step and a four weeks prediction horizon, the problem consists of 4234 continuous variables, 456 binary variables and 5701 constraints. The computations were performed on a Fujitsu Desktop with two $2.13 \mathrm{GHz}$ processors.

The result of the optimization is shown in Fig. 5. A set of simulations are performed using the high-fidelity reservoir model implemented in the state-of-the-art reservoir simulator (SENSOR) with the optimal shut-in times retrieved from the optimization with the proxy model. Comparing these two results, i.e. the red and the blue line in Fig. 5 , we observe only small discrepancies between the result from optimization with the proxy model and the corresponding simulations using the high-fidelity model, except from a more prominent discrepancy during the first two time-steps. The latter may be due to reservoir pressure depletions prior to the optimization, i.e. small variations in the initial conditions of the proxy and the SENSOR model. The result is further compared against a naive approach, where we choose a constant shut-in time of one week each time the wells reach the minimum rate $q_{g c}$. The

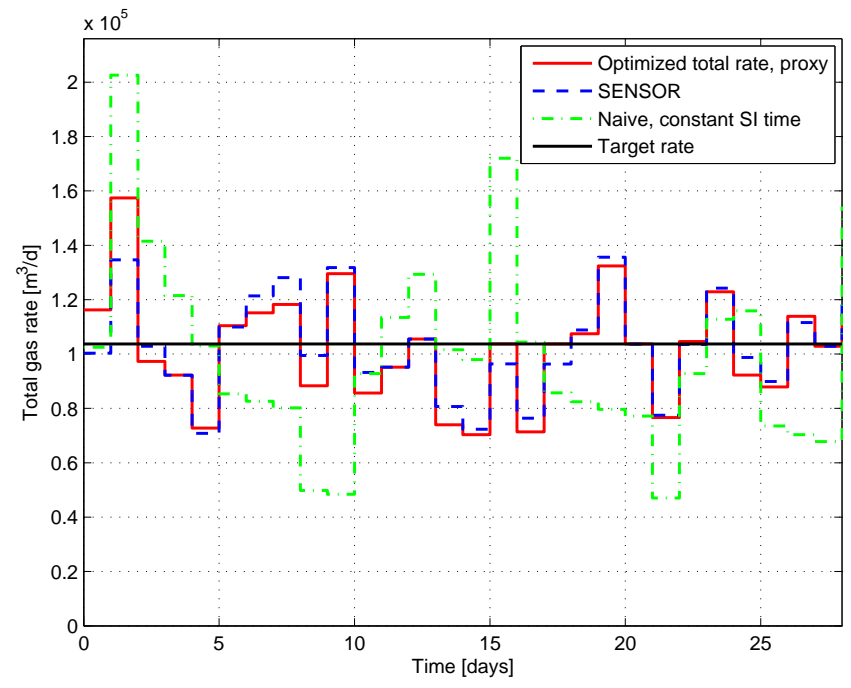

Fig. 5. Total gas rate from eight shale-gas wells subject to a specified target rate. The naive approach is implemented with a predefined constant shut-in (SI) time of one week.

naive shut-in strategy results in a more erratic rate than when the wells are scheduled with respect to the target rate, and the cumulative production is $4.1 \%$ less when the wells are shut in with the naive approach compared to the optimized shut-in times. Note that the constraints (28) and (30) are the minimum gas rate and the minimum shut-in time, respectively. Hence, the optimized shut-in times may lead to shut-ins at rates slightly higher than $q_{g c}$ and for durations longer than $\tau$ in order to track the target rate in (22) as tight as possible.

\section{DISCUSSION}

The two main contributions in this paper are the design and tuning of a shale-gas proxy model and the formulation of the optimization problem for scheduling of multi-well pads. The simple proxy model fits the high fidelity model well during the transients following the shut-ins, while it looses some accuracy in the pseudo steady-state region before the well is once more shut in. Including a transition region to modify the transmissibilities between the low permeability matrix and the high permeability fractured region was important to achieve good tuning results.

The proposed optimization problem finds optimal shutin times for the target-rate tracking problem, and the formulation has several merits: the penalty parameters can be selected in terms of a gas sales contract, or they can be selected to get as tight target-rate tracking as possible. Secondly, the formulation makes it straight forward to include additional operational constraints such as the minimum shut-in time for each well shut-in. The MILP model, in contrast to a corresponding MIQP formulation, lends itself to the use of robust and well-developed MILP solvers [Lodi, 2010], and the search for the optimal solution can be terminated whenever an acceptable duality gap is achieved. Still, there are several challenges with the problem formulation. For certain problem configurations in terms of initial conditions of the wells, the prediction horizon, the minimum shut-in time $\tau$ and the corresponding target rate, the optimizer is unable to converge to 
feasible solutions within 12 hours, except from the trivial solution of keeping all wells closed once the minimum rate $q_{g c}$ is reached. These observed convergence problems are related both to sensitivity of the initial well conditions, but generally also to the scalability of the problem since the number of constraints and integer variables increases rapidly if wells are added or the prediction horizon is extended.

The optimization problem is difficult in the sense that it is based on switching wells on and off to meet a target rate. Hence it may be, almost certainly, impossible to meet the target rate exactly, and the problems therefore terminate with relatively large duality gaps. Adding more wells makes the tracking tighter at the cost of the numerical efficiency. From the result in Fig. 5, it is observed that the optimal shut-in times result in a total production rate that varies quite a lot around the target rate. However, the total rate from the considered pad will just be one out of several multi-well pads producing gas to a shale-gas processing plant. The variations in the rate compared to the target rate may therefore be equalized by production rates from other pads, and this way produce a total gas rate to the processing plant that is close to a given multipad target rate. A viable approach for solving this type of large-scale plantwide control is the use of a decomposition approach similar to Gunnerud and Foss [2010].

One alternative production strategy to the proposed optimization of shut-in times, is to let the wells produce until liquid slugs or sharp drops in the production curve are observed and then shut the wells in for a desired time. The drawback with this approach is that its optimal shutin times are unknown and may be time varying. Another strategy would be to apply a constant, predefined shut-in time each time the rate falls below the critical rate $q_{g c}$. This leads to a cyclic shut-in scheme for each well without any consideration of well interactions or target-rate tracking. This type of naive shut-in scheme was implemented and compared with the optimized shut-in times in Fig. 5 , and observed to give a significantly more erratic total rate and slightly lower cumulative production. Rahmawati et al. [2009] implemented a similar type of cyclic shut-in strategy on a model of a hydraulic fractured well connected to a gas compressor. By optimizing the constant shut-in time, they observed that these types of wells could be shut in approximately $40 \%$ of the total production time without significant reduction in the total production. Extending the cyclic strategy to shut-ins with variable duration with respect to a production target should therefore be a sound approach. Note that in practice it may be necessary to shut in the wells slowly to reduce the pressure gradients arising from sudden shut-ins. This may be included in the specification of the minimum shut-in time $\tau$.

\section{CONCLUSIONS}

For the majority of active shale-gas plays, an extensive drilling program is applied to prevent decline in the field's total production rate. As a supplement to commissioning and drilling of a large number of new wells, the production optimization scheme proposed and tested in this paper is a possible novel direction for enhanced utilization of shalegas wells that are in a late-life phase and producing at low gas rates.

\section{REFERENCES}

J. H. Abou-Kassem, S.M. Farouq Ali, and M. Rafiq Islam. Petroleum Reservoir Simulation, A Basic Approach. Gulf Publishing Company, 2006.

H. Al Ahmadi, A. Almarzooq, and R. Wattenbarger. Application of Linear Flow Analysis to Shale Gas Wells - Field Cases. In SPE Unconventional Gas Conference, Pittsburgh, Pennsylvania, USA, 2010. SPE 130370-MS.

R. Al-Hussainy, H.J. Ramey Jr., and P.B. Crawford. The flow of real gases through porous media. Journal of Petroleum Technology, 18(5):624-636, 1966.

K. Aziz and A. Settari. Petroleum Reservoir Simulation. Applied Science Publishers Ltd, 1979.

C. L. Cipolla, E. P. Lolon, J. C. Erdle, and B. Rubin. Reservoir Modeling in Shale-Gas Reservoirs. In SPE Eastern Regional Meeting, Charleston, West Virginia, USA, 2009. SPE 125530-MS.

C. A. Floudas. Nonlinear and Mixed-Integer Optimization: Fundamentals and Applications. Oxford University Press, 1995.

V. Gunnerud and B. Foss. Oil production optimization - A piecewise linear model, solved with two decomposition strategies. Computers \& Chemical Engineering, 34(11): 1803-1812, 2010.

M. Karimi-Farad, L. J. Durlofsky, and K. Aziz. An Efficient Discrete-Fracture Model Applicable for GeneralPurpose Reservoir Simulators. SPE Journal, 9(2):227236, 2004.

D. L. Katz and R. L. Lee. Natural Gas Engineering. McGraw-Hill Publishing Company, 1990.

Andrea Lodi. Mixed integer programming computation. In 50 Years of Integer Programming 1958-2008, pages 619-645. Springer Berlin Heidelberg, 2010.

S. D. Rahmawati, C. H. Whitson, and B. Foss. Optimal Production Strategy for Stranded Tight-Gas Reservoirs. In Seminar on stranded gas including low permeability reservoirs, Yogyakarta, Indonesia, 2009.

R. Stefik and K. Paulson. When Unconventional Becomes Conventional. Journal of Canadian Petroleum Technology, 50(11):68-70, 2011.

R. P. Sutton, S. A. Cox, and R D Barree. Shale Gas Plays : A Performance Perspective. In SPE Tight Gas Completions Conference, San Antonio, Texas, USA, 2010. SPE 138447-MS.

R.G. Turner, M.G. Hubbard, and A.E. Dukler. Analysis and Prediction of Minimum Flow Rate for the Continuous Removal of Liquids from Gas Wells. Journal of Petroleum Technology, 21(11):1475-1482, 1969.

J.E. Warren and P.J. Root. The Behavior of Naturally Fractured Reservoirs. SPE Journal, 3(3):245-255, 1963. 